\title{
Improving medical induction in obstetrics and gynaecology
}

\author{
Eve Allen, Edward Palmer, Jilly Lloyd
}

University Hopsital Lewisham

\begin{abstract}
We present a year long quality improvement project to bring a new induction programme to the obstetrics and gynaecology (O\&G) department of University Hospital Lewisham (UHL). Aimed at non-speciality junior doctors, including general practice and foundation trainees, the induction programme has sought to improve the quality of care delivered and experience of these transiting junior doctors.

We have demonstrated a readily implementable and sustainable programme that requires only modest input of time from senior trainees (ST3+) periodically throughout the year. We have highlighted the specific need for senior consultant investment in the success and sustainability of such a project. We have demonstrated improvement of learning outcomes $(p=0.01)$ in junior doctors undertaking the induction programme at Kirkpatrick's hierarchy level 2.
\end{abstract}

\section{Problem}

Every four months there are at least eight junior doctors rotating through the O\&G department at UHL. These doctors encompass a board range of non-speciality foundation and general practice trainees. Most have no post graduate training in $O \& G$, and indeed, many will have last encountered this field during medical school. All have immediate on-call responsibility including the immediate management of acute gynaecology cases arriving through accident and emergency.

Prior to this intervention, departmental induction consisted of a meeting with a nominated consultant and an informal tour of the departmental areas within the hospital. Anecdotally, junior doctors on their O\&G rotation felt that they were underprepared for their job. We identified a need to create a structured induction programme to better prepare these junior doctors for their role and create a culture of inclusivity within the department.

\section{Background}

In 2012 Sir Bruce Keogh, medical director of the NHS, offered an open letter to all final year medical students in the UK detailing his hopes for an improvement in induction prior to their starting clinical practice.[1] This detailed a minimum of four days induction, with a focus on: improved familiarity with the new working environment, outlining responsibilities for safe and effective patient care, including how to seek supervision, improved familiarity with IT systems and procedures, and understanding what their roles and responsibilities are, as well as their limitations.

In discussion with our colleagues, we have found the response from NHS trusts to Sir Bruce Keogh's letter has generally been a worrying move towards using these four mandated days to fulfill the trusts mandatory and statutory training requirements. This is no doubt a far cry from Sir Bruce Keogh's original intentions.
Very little has been said with regards to department specific induction beyond the transition form medical student to junior doctor. This is despite the frequent use of Foundation Year 2, and general practice trainee doctors within many specialties, whom are often asked to conduct themselves in the same capacity as their specialty training senior house officer $(\mathrm{SHO})$ counterparts, despite significantly less training. The Royal College of Obstetricians and Gynaecologists (RCOG) states, 'inductions need to be comprehensive, ensuring that junior doctors are aware of the structures and procedures in place within the hospital; from operating the internal telephone system to who to approach in an emergency.'[2] In short, induction of junior doctors is a critical component of delivering safe and effective care.

\section{Baseline measurement}

A focus group was conducted with junior doctors currently and recently working in O\&G at UHL. The interview revealed a number of key themes. Junior doctors were generally dissatisfied with the existing departmental induction, specifically, the lack of dedicated time and resources. This led to concerns that the junior doctors felt under prepared and under supported for their new roles. There was an almost universal tone of anxiety surrounding the technical ability of junior doctors to perform and evaluate intimate examinations.

Further directed questioning yielded a number of more specific points that the trainees felt would be useful to include in future inductions including: focussed teaching on intimate examination, review of cervical/vulval pathology, clarification on roles and responsibilities within the department, basic maternity do's and don'ts, review of antenatal care including red flags for "do not miss" presentations and a practical overview of ward duties.

\section{Design}

A core team (the authors) was created to develop the induction programme including a foundation year two trainee and two ST7 
BMJ Quality Improvement Reports

O\&G speciality trainees. Consultants, senior nurses, and midwives were consulted as to what they felt was necessary content for a successful induction programme.

A basic programme was devised with key themes identified from the RCOG, national Commissioning for Quality and Innovation (CQUIN) targets and views expressed by existing junior doctors. Key themes included: departmental structure, whom to seek for support and advice, the rota, roles and responsibilities whilst on call, safety issues such as prescribing in pregnancy, venous thromboprophylaxis CQUIN targets, lectures on common obstetric and gynaecology emergencies, a review of intimate examinations, and a tour of the department.

The pilot induction programme was to be delivered by the current cohort of ST3-7 trainees. Each trainee was allocated a short segment of the day and assigned a topic of their choice. They were responsible for writing the lesson material and learning objectives for this session. These presentations were subsequently collected for future induction use, negating the need to rewrite the material at each new intake. By approaching a broad range of ST3-7 trainees to deliver the induction, we hoped to spread the burden of work whilst reducing rota conflict on the day.

We developed online feedback to assess junior doctor satisfaction undertaking the pilot induction. This also allowed feedback to be forwarded to trainees teaching on the induction, providing evidence for their portfolios and further motivation to assist with the induction. Feedback would help to inform on developing future iterations of the induction.

\section{Strategy}

PDSA 1 - Induction August 2013

In order to address the logistics of removing several trainees from the rota to deliver the induction programme, senior approval was needed. We approached the consultant co-ordinating the rota to allocate the first day of the rotation to the induction. Senior trainees were sequentially relieved of their duties throughout the day in order to deliver their session. The induction was organised as a series of lecture style sessions, tours, and practical hands on sessions. A brief timetable is provided here:

\section{- Registration and team introduction (15 minutes)}

- Tour of womens' health department and introduction to department heads (30 minutes)

\section{- Abdominal and intimate examination and the speculum (30 minutes)}

- The basics of cardiotocograph interpretation (30 minutes)

- Morning break (30 minutes)

- Common antenatal problems (1 hour)
- Drugs and prescribing in maternal populations (20 minutes)

- "Special circumstances" in labour (20 minutes)

- Lunch (30 minutes)

- Common postnatal problems (40 minutes)

- Sepsis (20 minutes)

- Early pregnancy (30 minutes)

- Acute gynaecological problems (1 hour)

- Afternoon break (15 minutes)

- Departmental admin, audits and agendas (20 minutes).

Despite a dependence on a broad range of individuals, only one session was unable to run as the senior trainee delivering that session was detained with clinical duties. All attendees were asked to complete a structured feedback of the session immediately following and two months into the rotation. We were able to gain insight into what the attendees thought of the induction both at the time and later on once they had time to appreciate the requirements of their post. Details of this feedback can be found in the results and appendix section. We used this feedback to inform and develop the next induction.

PDSA 2 - Induction December 2013

The programme was handed over to a new starting senior trainee. Lectures were transferred from the previous induction day, so that minimal further work was required to develop the course materials. Despite the success of the previous induction day, support from more senior faculty was lost and the induction was shortened to a half day delivered by a single senior trainee. Whilst feedback was still generally positive, overall satisfaction scores were appreciably lower. We feel that this was a reflection of the loss of senior support for the programme.

PDSA 3 - Induction April 2014

Based on the lessons learnt from the previous improvement cycle, a specific focus was placed on regaining support from the senior faculty and focussing on the organisational running of the day. The induction programme was rearranged as a whole day programme with a varied team delivering the lectures and tour. The rota was redesigned to allow maximum attendance on the first day of the post. In order to demonstrate the need for the induction, and provide evidence of its benefit, a pre/post course single best answer (SBA) test was developed to evaluate speciality specific knowledge acquisition, in addition to post induction feedback. This provided evidence to support the time away from the rota for future induction days. Additional hands on sessions were included with simulated obstetric emergencies, gynaecology examinations, and a 'cervix gallery' addressing some of the concerns raised by trainees in the focus group. A brief timetable is provided here : 


\section{BMJ Quality Improvement Reports}

- Arrival and completion of pre course SBA (30 minutes)

- Tour of early pregnancy assessment unit (20 minutes)

- Departmental tour (20 minutes)

- "What we expect of you, what we don't expect of you", consultant lead session (20 minutes)

- Morning break (10 minutes)

- Acute gynaecological problems (2 hours)

- Gynaecology do's and don'ts

- Lunch (30 minutes)

- Abdominal and intimate examination, the speculum and cervical atlas (2 hours)

- Close and post course SBA.

\section{Results}

At the end of each induction, qualitative feedback was collected using a nine-point Likert scale on a number of domains. In addition, we asked for free text feedback on how the trainees felt the induction could be improved. A summary of these results can be found in the appendix.

The mean result for the pre/post SBA demonstrated a significant improvement from $68 \%$ correct (pre course) to $88 \%$ correct (post course). A two tailed paired student's $\mathrm{T}$ test was used to identify significance $(t=3.49, p=0.01, n=8)$.

See supplementary file: ds3665.pdf - "Data collected"

\section{Lessons and limitations}

Whilst the programme was written and delivered by a nonconsultant faculty, it was evident that without senior support for the programme, it was not achievable. We identified support from the consultant in charge of rota co-ordination who had the authority to allow multiple trainees of the rota for the induction day.

Continued sustainability and improvement relies on a new coordinator to be identified each year with appropriate senior level support to facilitate time off the rota and resources. The overall value of induction has been continually underestimated by senior staff, as demonstrated by the reduced engagement for PDSA cycle 2. Delegation of induction duties to a specific named senior trainee for the year allows a constant point of continuity through which the induction programme can be sustainably developed. This trainee can engage in formal teaching, management and leadership skills, all acting as powerful motivators for a trainee to take on the responsibility of co-ordinating the induction programme.
When we identified the importance of practical sessions, high fidelity training on gynaecology training associates (the gold standard on teaching intimate examinations) was investigated. However, the cost of using this service was prohibitively expensive at around £200 per trainee. As such, we developed the programme to use simulation models already available in the department allowing a more cost effective induction. As the profile of the induction improves, it may be possible to revisit this funding issue to further improve the experience of trainees.

\section{Conclusion}

In recent years, both the Francis report and the Keogh review have highlighted the importance of junior doctor inclusion into the medical team. Junior doctors are key to the delivery of high quality and safe care. By creating a trainee centred induction into $O \& G$ for nonspeciality junior doctors, we have started to create a culture of inclusivity, support, and training amongst the O\&G faculty at UHL.

Induction feedback has generally been positive, and continues to improve as we make adjustments to the program based on previous session feedback. As a more formal evidence for the educational value of the course, we have demonstrated an improvement in learning outcomes in trainees that undertook the induction at Kirkpatrick hierarchy of learning level 2.

Importantly, this programme has focussed on long term sustainability by creating a policy of handing over the co-ordination of the induction to a named senior trainee for the duration of their training at UHL. By passing on session materials from one induction to the next, the initial work required to deliver the programme has been maximally reduced, so the focus can be on organising and managing the induction day itself.

As a direct result of the induction programme, junior doctors on rotation in O\&G at UHL feel better prepared for their responsibilities, feel that their practice is safer and better understand their place within the department.

\section{References}

1. Department of Health. Lives will be saved as junior doctors shadow new role. (2012, June 25). Retrieved from https://www.gov.uk/government/news/lives-will-be-saved-asjunior-doctors-shadow-new-role.

2. RCOG. (2009). RCOG statement on the paper "Early InHospital Mortality following Trainee Doctors' First Day at Work" http://www.rcog.org.uk/what-we-do/campaigning-andopinions/statement/rcog-statement-paper-'early-hospitalmortality-followi.

\section{Declaration of interests}

Nothing to declare.

\section{Acknowledgements}


BMJ Quality Improvement Reports

Many thanks to Mahishee Mehta and Anne Billett for their support. 